\title{
1 BRD9-containing non-canonical BAF complexes safeguard cell identity and
}

\section{2 prevent reprogramming}

3 Kenan Sevinç $c^{1,7}$, Gülben Gürhan Sevinç ${ }^{1,7}$, Ayşe Derya Cavga ${ }^{1,2}$, Martin Philpott ${ }^{3}$, Simge

4 Kelekçi ${ }^{1}$, Hazal Can ${ }^{1}$, Adam P. Cribbs ${ }^{3}$, Enes Sefa Ayar ${ }^{1}$, Dilşad H. Arabac1 ${ }^{1}$, James E.

5 Dunford ${ }^{3}$, Ata B. Demir ${ }^{1}$, Logan H. Sigua ${ }^{4}$, Jun $\mathrm{Qi}^{4}$, Udo Oppermann ${ }^{3,5,6,8}$, Tamer T. Onder ${ }^{1,8, *}$

$7 \quad{ }^{1}$ School of Medicine, Koç University, Istanbul, Turkey.

$8{ }^{2}$ Biostatistics, Bioinformatics and Data Management Core, KUTTAM, Koç University, Istanbul, 9 Turkey.

$10 \quad{ }^{3}$ Botnar Research Centre, Oxford NIHR BRU, University of Oxford, Oxford, UK.

$11{ }^{4}$ Department of Cancer Biology, Dana Farber Cancer Institute, Boston, MA, USA.

$12{ }^{5}$ Centre for Medicine Discovery, University of Oxford, Oxford, UK.

$13{ }^{6}$ Oxford Centre for Translational Myeloma Research, University of Oxford, OX3 7LD, UK

$14{ }^{7}$ These authors contributed equally.

$15 \quad{ }^{8}$ These authors contributed equally.

$16 *$ Correspondence: tonder@ku.edu.tr

\section{Abstract}

Epigenetic reprogramming requires extensive remodeling of chromatin landscapes to

20 silence cell-type specific gene expression programs. ATP-dependent chromatin-remodeling

21 complexes are important regulators of chromatin structure and gene expression; however, the role

22 of Bromodomain-containing protein 9 (BRD9) and the associated ncBAF (non-canonical BRG1-

23 associated factors) complex in reprogramming remains unknown. Here, we show that genetic

24 suppression of BRD9 as well as ncBAF complex subunit GLTSCR1, but not the closely related

25 BRD7, increase the efficiency by which induced pluripotent stem cells (iPSCs) can be generated

26 from human somatic cells. Chemical inhibition and acute degradation of BRD9 phenocopied this

27 effect. Interestingly, we find that BRD9 is dispensable for establishment and maintenance of 
28 human pluripotency but required for mesendodermal lineage commitment during differentiation.

29 Mechanistically, BRD9 inhibition downregulates somatic cell type-specific genes and decreases

30 chromatin accessibility at somatic enhancers. Collectively, these results establish BRD9 as an

31 important safeguarding factor for somatic cell identity whose inhibition lowers chromatin-based

32 barriers to reprogramming.

Introduction erase somatic cell identity and reprogram the cells to a pluripotent state $(1,2)$. In doing so, reprogramming factors reset the entire epigenetic landscape established throughout development (3). The varying and low efficiencies of this process point to the presence of intrinsic somatic barriers to cell fate conversions. Several chromatin factors such as DOT1L methyltransferase (4),

41 histone chaperone CAF-1 (5), BET family proteins (6), RNA Pol II regulator RPAP1 (7), SUMO

42 modification (8), chromatin regulator FACT (9) and CBP/EP300 bromodomains (10) have

43 emerged as potent barriers to reprogramming and act mainly by safeguarding pre-existing gene 44 expression programs. Inhibition of these factors greatly facilitate reprogramming of a wide range 45 of cell types (11). Discovery of additional safeguarding factors will likely yield important insights 46 into chromatin-based mechanisms that maintain cell identity and restrict cell plasticity.

ATP-dependent chromatin remodeling complexes evict, exchange and space nucleosomes

48 driven by the hydrolysis of ATP (12). Chromatin remodelers can facilitate transcriptional

49 activation or repression based on the genomic location they bind to and additional chromatin 50 factors they recruit (13-15). Among these, NuRD, INO80 and SWI/SNF complexes have been 
51 shown to modulate reprogramming in a variety of contexts (16-19). For example, overexpression

52 of BAF complex subunits Smarca4 and Smarcc1 enhances murine somatic cell reprogramming by

53 facilitating binding of Oct4 to its gene targets (18). In contrast, BAF complex subunits, Smarca2

54 and Smarcc2, have shown to be barriers in this context through upregulation of Stat3 and its target

55 genes (20). Suppressing these somatic BAF subunits have been shown to activate the pluripotency

56 circuit (20). These studies point to regulatory roles for different ATP-dependent chromatin

57 remodeling complexes in various reprogramming frameworks.

Non-canonical BAF (ncBAF) complex is a recently identified SWI/SNF complex that lacks

SMARCE, SMARCB, ARID1 and DPF but includes specific subunits such as BRD9 and

GLTSCR1/L (21-25). BRD9 binds to enhancer regions in a cell type-specific manner and

61 inhibition of its bromodomain leads to apoptosis in acute myeloid leukemia cells (26). Similarly,

62 BRD9 inhibition leads to decreased cell proliferation, G1-arrest and apoptosis in rhabdoid tumor

63 cells (24). In mouse embryonic stem cells (mESCs), ncBAF has been shown to co-localize with

64 key regulators of naive pluripotency and BRD9 bromodomain inhibitors abolish naïve

65 pluripotency by disrupting ncBAF's recruitment to chromatin (27). These studies suggest that

66 BRD9 is important for regulating cell identity and survival. However, the role of BRD9 and the

67 ncBAF complex in somatic cell reprogramming remains unknown. In this study, we addressed this

68 question using a combination of chemical and genetic tools in somatic cells and revealed an

69 important role for BRD9 in safeguarding cell identity in the context of human reprogramming.

70 Results

72 Genetic suppression of ncBAF-specific subunits increases reprogramming efficiency 
To investigate the role of ncBAF complex in reprogramming, we employed two genetic

74 loss-of-function approaches. First, knockdown of $B R D 9$ using 2 independent shRNAs increased

75 reprogramming efficiency 2-fold (fig. S1, A and B). On the other hand, suppression of BRD9

76 paralog and PBAF-specific subunit, $B R D 7$, had no effect on reprogramming even though it was

77 efficiently knocked-down (fig. S1, A and C). In the second approach, we utilized sgRNAs and

78 Cas9 to knock-out BRD9 and then test the reprogramming efficiency of the resulting cells.

79 Consistent with the shRNA data, knock-out of $B R D 9$, but not $B R D 7$, boosted reprograming

80 efficiency up to 3-fold compared to control sgRNA expression (Fig. 1, A, B and C). Based on these

81 results, we hypothesized that among the various BAF complexes in somatic cells, BRD9-

82 containing ncBAF complex is a specific barrier for reprogramming. To test this notion, additional

83 ncBAF specific subunits Glioma tumor suppressor candidate region gene 1 (GLTSCR1) or its

84 paralog GLTSCR1-like (GLTSCR1L) were targeted by sgRNAs in human fibroblasts (21).

85 Knocking out GLTSCR1 increased reprogramming efficiency up to 4-fold for 3 sgRNAs out of 4

86 tested compared to control sgRNA expression (Fig. 1D). Targeting GLTSCR1 paralog,

87 GLTSCRIL, had no effect on reprogramming even though T7 endonuclease assay confirmed indel

88 formation at this locus (Fig. 1D and fig. S1D). These results show that ncBAF complex members

89 act as barriers to human somatic cell reprogramming.

\section{Bromodomain inhibition and degradation of BRD9 facilitate reprogramming}

To confirm the role of BRD9 in somatic cell reprogramming, we next employed 3

92 structurally different inhibitors LP99, BI-7273 and I-BRD9 all of which selectively target the

93 bromodomain of BRD9 and block its interaction with acetylated lysines (28-30) (Fig. 2A). All

94 three BRD9 bromodomain inhibitors significantly increased reprogramming efficiency of human 
95 fibroblasts to iPSCs up to two-fold at concentrations of 1 and $3 \mu \mathrm{M}$ (Fig. 2B). Importantly, I-BRD9

96 treatment had an additive effect with the inhibition of DOT1L, a potent reprogramming enhancer

97 that we had previously identified (4). Combined inhibition of BRD9 and DOT1L led to a

98 remarkable 5-fold increase in the number of iPSCs generated from human fibroblasts (fig. S2A).

99 Emerging iPSC colonies in both control and BRD9 bromodomain inhibitor-treated cultures

100 exhibited canonical characteristics of pluripotency such as retroviral transgene silencing and

101 expression of pluripotency-specific markers OCT4, SSEA4 and NANOG (fig. S2, B and C). When

102 injected into immunodeficient mice, iPSCs derived from I-BRD9-treated fibroblasts were able to

103 form teratomas containing differentiated cells from all three germ layers (fig. S2D). These results

104 show that inhibition of BRD9 bromodomain enhances human somatic cell reprogramming.

Next, we took advantage of a recently described PROteolysis Targeting Chimera

106 (PROTAC) targeting BRD9, dBRD9, to acutely deplete this protein (31). Time-course

107 experiments showed that treatment with dBRD9 can dramatically decrease BRD9 protein levels

108 for up to 72 hours without any effects on the closely related BRD7 (Fig. 2C). We observed that

109 reprogramming efficiency increased up to 2-fold compared to control treatment even at the lowest

110 concentration of $0.1 \mu \mathrm{M}$ dBRD9 tested (Fig. 2D). A similar phenotype was observed in episomal

111 plasmid-based reprogramming of an additional human dermal fibroblast line, indicating that BRD9

112 inhibition enhances iPSC generation independent of reprogramming strategy or cell line used (fig.

$113 \mathrm{~S} 2 \mathrm{E})$. Taken together, these results show that bromodomain inhibition or acute degradation of

114 BRD9 increases human somatic cell reprogramming efficiency.

115 To understand how BRD9 inhibition promotes iPSC generation, we first determined when

116 in the reprogramming process its inhibition has the maximal effect. A time-course treatment 
117 experiment with I-BRD9, dBRD9 and DMSO control was performed at different time-windows to

118 evaluate which stage of reprogramming is responsive to BRD9 inhibition (Fig. 2E). Inhibition or

119 degradation of BRD9 had the most effect on reprogramming efficiency when applied during the

120 first 6 days after OSKM expression. As there were no further increases in the number of iPSCs

121 with longer periods of chemical treatments, we concluded that BRD9 is a barrier for the initial

122 stage of reprogramming (Fig. 2, F and G). In addition, the percentage of emerging TRA-1-60

123 positive cells on day 6 of reprogramming, as assessed by flow cytometry, was significantly higher

124 in BRD9 bromodomain inhibitor-treated cultures compared to controls at this early time-point

125 (Fig. 2H and fig. S2F). Taken together, these results indicate that the initial stage of reprogramming

126 is most sensitive to BRD9 inhibition and that even a transient BRD9 inhibition is sufficient to

127 increase the efficiency of human iPSC generation.

BRD9 inhibition and degradation enable iPSC generation without KLF4 and c-MYC.

We previously reported that inhibition of somatic barriers to reprogramming can enable

130 human iPSC generation with fewer Yamanaka factors $(4,10)$. To investigate if BRD9 inhibition

131 may have a similar effect, we carried out reprogramming with only OSK or OS. In both

132 circumstances, reprogramming efficiency was increased with BRD9 inhibition (Fig. 3, A and B).

133 PCR with vector-specific primers validated the absence of KLF4 and MYC transgenes in genomic

134 DNAs of iPSCs derived from OS-transduced fibroblasts (Fig. 3C). Importantly, iPSCs derived by

135 OS transduction could be stably propagated and exhibited pluripotency characteristics such as

136 silencing of retroviral transgenes, expression pluripotency markers such as OCT4, SSEA4 and

137 NANOG and ability to form teratomas containing differentiated cells from all three germ layers 
138 (Fig. 3, D, E and F). These results show that BRD9 inhibition can enable iPSC generation with

139 fewer exogenous transcription factors.

140 BRD9 is dispensable for human pluripotency induction and maintenance but required for mesendedorm differentiation.

While small molecules allow for transient inhibition or degradation of BRD9 during

143 reprogramming, sgRNA expression in fibroblasts may result in a permanent knockout in the

144 resulting iPSCs. We therefore wished to determine whether the TRA-1-60-positive colonies

145 generated from fibroblasts expressing $B R D 9$ sgRNAs have a complete absence of BRD9 protein.

146 iPSC colonies derived from control and $B R D 9$ sgRNA expressing fibroblasts were expanded in

147 culture and BRD9 protein levels were examined (Fig. 4A). 9 iPSC lines out of 14 generated from

148 BRD9 sgRNA expressing fibroblasts did not express BRD9 at all, suggesting a homozygous

149 knockout. 4 iPSC lines had reduced protein expression suggestive of a heterozygous knockout and

1501 iPSC colony retained wildtype levels of BRD9 (Fig. 4B). Complete knock-out clones robustly

151 expressed OCT4, NANOG and SSEA4 similar to control iPSC lines (fig. S3A). The observation

152 that the majority of the expanded clones expressed no BRD9 protein while exhibiting hallmarks

153 of pluripotent stem cells suggests that loss of BRD9 is dispensable for human iPSC generation and

154 propagation.

To further characterize the pluripotency BRD9 knock-out hiPSCs, we investigated their

156 differentiation capacity. BRD9-knockout iPSCs were able to form teratomas containing cells from

157 all three germ layers (Fig. 4C). However, we observed that tissues from mesoderm lineage were

158 less abundant in histological sections of teratomas generated by BRD9-knockout iPSCs. This

159 observation led us to hypothesize that BRD9 may have a role in mesoderm differentiation. To test 
160 this, we differentiated two independent iPSC lines generated from two healthy donors into

161 mesendoderm with WNT agonist CHIR99021 (32) and in the presence of small molecules

162 targeting BRD9. Expression of TBXT, EOMES and MIXL1, well-established markers for

163 mesendoderm, decreased upon BRD9 inhibition and degradation (Fig. 4D, fig. S3F). On the other

164 hand, exit from pluripotency as judged by expression of POU5F1, SOX2 and NANOG was not

165 affected by BRD9 inhibition (Fig. 4D, fig. S3F). These results suggest that BRD9 is important for 166 mesendodermal lineage commitment of human pluripotent stem cells.

Naïve mouse ESCs have been shown to be sensitive to BRD9 bromodomain inhibition when grown in serum and Lif (27), therefore we wished to further investigate the role of BRD9 in

169 human pluripotent stem cells. We made use of an OCT4-GFP reporter human iPSC line (33) to 170 monitor cell viability and self-renewal capacity upon BRD9 inhibition. BRD9 inhibitor or degrader

171 treatment for 48 hours did not change the proliferation rate nor the percentage of OCT4-positive

172 cells compared to controls (fig. S3, B, C and D). These results, in combination with the knockout

173 iPSC lines, suggest that BRD9 is not required to maintain human pluripotency despite it being

174 necessary for naïve pluripotency in the mouse (27). To specifically examine if the role of BRD9

175 in pluripotency acquisition is species-specific, we reprogrammed mouse embryonic fibroblasts in

176 the presence of small molecules targeting BRD9. BRD9 inhibition and degradation did not 177 increase murine somatic cell reprogramming; in fact, we observed a modest decrease in efficiency 178 with the degrader (fig. S3E). These results show that BRD9, in contrast to its function in murine 179 naive PSCs, is not required for the induction and maintenance of human pluripotency.

\section{BRD9 maintains somatic-specific gene expression and enhancer accessibility}


Given that BRD9 inhibition is most effective in early reprogramming, where

184 this end, we performed mRNA-sequencing from fibroblasts treated with BI-7273, I-BRD9,

$185 \mathrm{dBRD} 9$ as well as those expressing Cas9 and BRD9 sgRNA. Small molecules targeting BRD9

186 differentially downregulated 928, 170, 577 genes (dBRD9, I-BRD9, BI-7273, respectively), of

187 which 70 were common to all treatments (fig. S4A). Gene ontology (GO) analysis of this common

188 set of genes revealed that they were highly enriched in cellular process linked to fibroblast identity

189 and function, such as epithelial-to-mesenchymal transition (EMT), extracellular matrix

190 components and adhesion (Fig. 5A). More broadly, Gene Set Enrichment Analysis (GSEA)

191 indicated epithelial-to-mesenchymal transition (EMT) gene sets were among the top most

192 negatively regulated gene sets upon I-BRD9 and dBRD9 treatments (Fig. 5B). This finding led us

193 to ask specifically whether the fibroblast expression program as a whole was downregulated upon

194 BRD9 inhibition. To test this notion, we performed GSEA using a fibroblast-related gene set (307

195 genes) that we generated based from gene expression profiles of human fibroblasts and their iPSC

196 derivatives (10). All BRD9 perturbations resulted in a highly significant downregulation of the

197 fibroblast-related gene set (Fig. 5C). In fact, the overall average expression values of genes in the

198 fibroblast-related gene set were significantly downregulated upon BRD9 inhibition (Fig. 5D). In

199 contrast, we did not observe positive enrichment of pluripotency-associated gene sets with any

200 BRD9 perturbation, indicating that BRD9 inhibition on its own does not activate the pluripotency

201 network (fig. S4B). Taken together, our transcriptomic analyses suggest that BRD9 acts as a barrier

202 to reprogramming by sustaining starting cell type-specific gene expression. 
To gain insight into how BRD9 functions to maintain expression of somatic-specific genes and test whether BRD9 has a role in maintaining chromatin accessibility at such loci, we performed ATAC-seq (Assay for Transposase-Accessible Chromatin using sequencing) in fibroblasts treated with I-BRD9 and dBRD9. Both inhibitors had no effect on accessible chromatin regions around promoters marked by overlap of H3K27ac and H3K4me3 (Fig. 5E). However, BRD9 inhibition or degradation reduced the accessibility of chromatin around putative active enhancers in fibroblasts as marked by overlap of $\mathrm{H} 3 \mathrm{~K} 27 \mathrm{ac}$ with $\mathrm{H} 3 \mathrm{~K} 4 \mathrm{me} 1$ (Fig. 5F). Importantly, such fibroblast-specific

211 enhancers start to lose accessibility upon OSKM expression, suggesting BRD9 inhibition 212 augments this process (fig. S4C) (34). These results indicate that BRD9 constitutes a barrier to 213 reprogramming by maintaining accessibility of active enhancers in the starting cell populations.

\section{$214 \quad M N 1$ and $Z B T B 38$ are BRD9 target genes that suppress reprogramming} transcriptional regulators $M N 1$ and $Z B T B 38$ (Fig. 6A). MN1 has not been implicated in somatic

217 cell reprogramming, but regulates palate development (35) and can act as co-factor for various 218 transcription factors such as retinoic acid receptor/retinoic $\mathrm{X}$ receptor (RAR/RXR) (36). It is also 219 implicated in transcriptional control of leukemic transformation in collaboration with DOT1L (37).

220 ZBTB38 is predicted to be a master regulator in fibroblasts and is controlled by a fibroblast specific

221 super-enhancer (38). We hypothesized that downregulation of these two factors soon after OSKM 222 expression is necessary for efficient reprogramming. To test this notion, we overexpressed MN1 223 or ZBTB38 along with OSKM in human fibroblasts which resulted in a significant impairment in 224 reprogramming efficiency (Fig. 6, B, C and D). Interestingly, ZBTB38 expression in fibroblasts 225 also significantly reduced the number of emerging TRA-1-60-positive cells on day 6 (Fig. 6E). 
Moreover, induction of pluripotency associated genes such as NANOG, LEFTY2, LIN28A were reduced at day 6 of reprogramming upon continued $Z B T B 38$ expression (Fig. 6F). These results BRD9 safeguards somatic cell identity and acts as a barrier to reprogramming in part by sustaining

229 the expression these two transcriptional regulators (Fig 6G).

\section{Discussion}

In this study, we investigated the role of BRD9 in human somatic cell reprogramming to

232 pluripotency via a combination of genetic and chemical perturbation approaches. Knockdown of

233 BRD9 via RNA interference or knockout via CRISPR/Cas9 increased human reprogramming

234 efficiency. Importantly, the contrasting effects of BRD9 and BRD7 inhibition on reprograming

235 efficiency indicate that the recently identified BRD9-containing ncBAF, but not BRD7-containing

236 PBAF, is a major barrier to reprogramming. This notion is supported by our finding that loss of an

237 additional specific member of ncBAF complex, GLTSCR1, has a similar positive effect on iPSC

238 formation. To acutely block BRD9 function, we took advantage of selective bromodomain

239 inhibitors and a PROTAC degrader, all of which significantly increased reprogramming efficiency

240 and enabled iPSC generation in the absence of KLF4 and cMYC. Taken together, these findings

241 demonstrate that BRD9-containing ncBAF complexes serve an important role in maintaining

242 somatic cell identity.

243 Interestingly, we find that BRD9 is dispensable for induction and maintenance of human

244 pluripotency. Fibroblasts expressing Cas9 and sgRNAs against BRD9 could efficiently generate

245 iPSCs that do not express any detectable BRD9 protein. Yet, such iPSC clones, and additional

246 iPSCs treated with compounds targeting BRD9, could be propagated and expanded while retaining

247 canonical properties of pluripotent stem cells. This is in contrast to the non-BRD9 containing ES-

248 specific BAF complexes which are required for pluripotency and self-renewal (39). Interestingly, 
naïve mESCs have been shown to lose self-renewal capacity and enter into a primed, epiblast-like transcriptional state upon BRD9 inhibition (27). Human iPSCs are considered to be in a primed

251 state (REF), our data indicate that BRD9 is dispensable for their maintenance. It is therefore likely 252 that BAF complexes other than ncBAF such as non-BRD9 containing ES-specific complexes 253 support human primed pluripotency. It is also worth noting that mouse and human reprogramming 254 systems may have distinct species-specific features in part due to chromatin organization $(40,41)$.

255 In fact, we observed that murine reprogramming was not enhanced upon BRD9 inhibition nor 256 degradation. Our findings are consistent with a recent study in which I-BRD9 were found not to 257 increase reprogramming efficiency from mouse embryonic fibroblasts (42). Collectively, these 258 observations point to species-specific roles for BRD9 in somatic cell reprogramming and 259 maintenance of pluripotency.

261 fibroblast-enriched genes which is accompanied by decreased chromatin accessibility across 262 putative active enhancers and cell-type specific super-enhancers. These results align with previous 263 studies which show BRD9 occupancy at distal enhancers $(26,43)$ and co-localization with CTCF 264 (23, 44). In addition, BRD9 have recently been shown to be crucial for maintaining cell-type 265 specific transcription programs in regulatory T-cells (45). We identified several key transcription 266 factors such as $M N 1$ and $Z B T B 38$ as BRD9 targets. Our functional data, along with recent studies, 267 establish these BRD9-regulated genes as important barriers to reprogramming (46).

269 small molecule inhibitors that can be used to regulate and direct cell fate changes in human somatic

270 cells. We also show that BRD9 inhibition can be combined with other modulators such as DOT1L 271 inhibitors to boost reprogramming efficiency. Importantly, inhibition of DOT1L-mediated H3K79 
272 methylation facilitates the generation of chemically induced pluripotent stem cells (ciPSCs) from

273 mouse somatic cells (47) and result in a permissive epigenome state which enables reprogramming

274 by alternative transcription factors (48). Identification of BRD9 as a safeguarding mechanism of

275 cell identity suggests that combinatorial perturbations which include BRD9 inhibitors can enhance

276 various reprogramming methods. Such approaches will likely lead to rapid silencing of the initial

277 somatic program and potentially be used to derive human ciPSCs as well as direct lineage-

278 converted cells with high efficiency.

279

$280 \quad$ Materials and Methods

281 Reprogramming assays. Human fibroblasts (dH1f) and reprogramming assays were performed

282 as described previously $(10,49)$. Unless stated otherwise in the text, I-BRD9, LP99, BI-7273 and

$283 \mathrm{dBRD} 9$ were used at final concentrations of $1 \mu \mathrm{M}, 3 \mu \mathrm{M}, 1 \mu \mathrm{M}$ and $0.3 \mu \mathrm{M}$, respectively, for the

284 first two weeks of reprogramming. EPZ-004777 was used at final concentration of $3 \mu \mathrm{M}$ for the

285 first week of reprogramming. HDF-A cells (ScienCell Research Laboratories, Catalog \#2320)

286 were reprogrammed with episomal vectors as described previously (50). For murine

287 reprogramming, 25000 mouse embryonic fibroblasts (MEF) cells were seeded at each well of 12

288 well plate. The next day, they were transduced by lentiviruses containing a single stem cell

289 cassette (51). Next day culture media was replenished with small molecules at the final

290 concentrations indicated for human somatic cell reprogramming conditions. At 5th day of

291 reprogramming, cells were transferred on inactivated MEFs. Next day and every other day until

292 14th day of reprogramming, culture media were replenished with mouse embryonic stem cell

293 media (20\% FBS, 1\% NEAA, 1\% Pen-Strep, 1\% L-glutamine, 55nM 2-Mercaptoethanol, 1000 
U/mL Leukemia Inhibitory Factor (LIF) (ESGRO, Catalog \#ESG1107) in Knockout DMEM

295 (Gibco, Catalog \#10829-018)).

296 iPSC culture and mesendodermal differentiation. Individual iPSC colonies generated from

297 BRD9 sgRNA and Cas9 expressing fibroblasts were manually picked and cultured on inactivated

298 MEFs with hESC media and ROCK inhibitor Y-27632 at a final concentration of $10 \mu \mathrm{M}$. After

299 a few passages clones were transitioned to feeder-free conditions on matrigel (Corning, Catalog

300 \#354277)-coated plates with MEF-conditioned hESC media. iPSCs used for CHIR99021-

301 induced mesendodermal differentiation experiments were picked from adult fibroblasts,

302 electroporated with non-integrative episomal plasmids and cultured on feeder-free conditions on

303 matrigel-coated plates with mTESR1 media $(50,52)$. When the cells reached 40-60\%

304 confluency, they were treated with DMSO, $1 \mu \mathrm{M}$ I-BRD9 or $0.1 \mu \mathrm{M}$ dBRD9 in $5 \mu \mathrm{M}$

305 CHIR99021 in fibroblast media for 48 hours.

306 Cloning. sgRNA and shRNA oligonucleotides (Table S1) were cloned into lentiCRISPR v2

307 (Addgene plasmid no. \#52961) and into pSMP vector (Addgene plasmid no. \#36394)

308 respectively. shRNA cloning was performed as previously described with XhoI and EcoRI (4).

309 In order to clone sgRNAs, lentiCRISPRv2 vector was digested with BsmBI enzyme and gel

310 purified with MN PCR-Clean up Kit. Extracted DNA was treated with AP. Top and bottom

311 guide RNAs were annealed with T4 Polynucleotide Kinase (3' phosphatase minus, NEB) and T4

312 DNA Ligase Buffer (NEB) in a thermal cycler with the following settings: $37^{\circ} \mathrm{C}$ for $30 \mathrm{~min}$,

$31395^{\circ} \mathrm{C}$ for $4 \mathrm{~min}$ and then ramp down to $25^{\circ} \mathrm{C}$ at $5^{\circ} \mathrm{C} / \mathrm{min}$. Annealed oligos were diluted to 1:200

314 and were used as inserts for ligating to digested lentiCRISPR v2 (50 ng) by T4 DNA Ligase

315 (NEB) in 10X T4 DNA Ligase buffer (NEB). Ligation reaction were incubated for at least 2

316 hours at room temperature. Ligation mix was vortexed and spun down. $50 \mu \mathrm{l}$ Stbl3 bacteria 
317 (NEB, Catalog: C737303) was mixed with $5 \mu$ of the ligation reaction and left on ice for at least

31815 minutes. Afterward, heat shock was applied at $42^{\circ} \mathrm{C}$ for 30 seconds in water bath. $150 \mu \mathrm{LB}$

319 was supplemented and cultured in $37^{\circ} \mathrm{C}$ at $225 \mathrm{rpm}$ in a shaker for 1 hour. The transformed

320 bacteria were spread on LB agar plate with ampicillin or carbenicillin.

321 Virus production and transduction. Virus production was performed as described previously

322 (10). Briefly, $2.5 \times 10^{6} 293$ T cells per $10-\mathrm{cm}$ dish were plated. The next day, cells were

323 transfected with $2.5 \mu \mathrm{g}$ viral vector, $0.25 \mu \mathrm{g}$ pCMV-VSV-G (Addgene plasmid no. 8454),

$3242.25 \mu \mathrm{g}$ psPAX2 (Addgene plasmid no. 12260) for lentivirus or pUMVC (Addgene plasmid no.

325 8449) for retroviruses using $20 \mu \mathrm{l}$ FuGENE 6 (Promega) in $400 \mu 1$ DMEM per plate.

326 Supernatants were collected $48 \mathrm{~h}$ and $72 \mathrm{~h}$ post-transfection and filtered through $45-\mu \mathrm{m}$ pore size

327 filters. Human fibroblasts were doubly transduced with either shRNA or CRISPR vectors in

328 consecutive days in the presence of $8 \mu \mathrm{g} / \mathrm{ml}$ protamine sulfate (Sigma). Transduced cells were

329 selected by puromycin at $1 \mu \mathrm{g} / \mathrm{ml}$.

330 Western Blot. Cell pellets were resuspended in cytosolic lysis buffer (10mM HEPES pH 7.9,

$33110 \mathrm{mM} \mathrm{KCl}, 0.1 \mathrm{mM}$ EDTA, 0.4\% NP-40, Protease Inhibitor (1X) (Roche)) and shaken on ice for

33215 minutes. After centrifugation at $3000 \mathrm{~g}$ at $40 \mathrm{C}$ for 3 minutes, pellets were washed with

333 cytosolic buffer again. Pellets were resuspended in nuclear lysis buffer (20mM HEPES pH 7.9,

$3340.4 \mathrm{M} \mathrm{NaCl}, 1 \mathrm{mM}$ EDTA, 10\% Glycerol, Protease Inhibitor (1X) (Roche)) and sonicated at

335 amplitude 40 for 10 seconds twice. Supernatant containing nuclear fragments was collected after

336 centrifugation at $15000 \mathrm{~g}$ at $40 \mathrm{C}$ for 5 minutes. Lysates were incubated at $950 \mathrm{C}$ for 15 minutes

337 with 4X laemmli buffer (BioRad) containing $\beta$-mercaptoethanol (BioRad). Boiled samples and

338 protein marker (Bio-rad, Catalog: 161-0374) were loaded on gel (BioRad, Catalog: 456-1084).

339 Proteins were transferred on PVDF membrane (BioRad, Catalog: 1620177) by Bio-Rad tans-blot 
340 turbo transfer system at mixed weight transfer setting and incubated in 5\% blotting grade blocker

341 (BioRad Catalog: 1706404) solution for 1 hour. Membranes were incubated with BRD9 antibody

342 (Active Motif, Catalog: 61537) at 1:1000 ratio, BRD7 antibody (Cell Signaling, Catalog:

343 D9K2T) at 1:1000 ratio and HDAC1 antibody (Santa Cruz, Catalog: sc-7872) at 1:500 ratio

344 overnight at 40C. Next day, membranes were washed with TBS-T for 15 minutes three times and

345 incubated with secondary antibody (Abcam, ab97051) for 1 hour at room temperature.

346 Membranes were washed with TBS-T for 15 minutes 3 times and incubated shortly with ECL

347 western blotting substrate (ThermoFisher) before imaging at LI-COR FC.

348 Genomic DNA PCR. Genomic DNA was extracted from cells with Nucleospin Tissue kit

349 (catalog no. 740952.50) following manufacturer's instructions. $200 \mathrm{ng}$ of genomic DNA was

350 amplified with DreamTaq DNA Polymerase (catalog no. K1081) and indicated primer pairs

351 (Table S1). OCT4, SOX2, KLF4 and c-MYC transgene-containing amplicons were amplified

352 with 30 cycles of $30 \mathrm{~s}$ at $95^{\circ} \mathrm{C}, 30 \mathrm{~s}$ at $54.5^{\circ} \mathrm{C}$ and $60 \mathrm{~s}$ at $72^{\circ} \mathrm{C}$. Amplicons containing

353 GLTSCR1L sgRNA-induced in-dels were amplified using a BIO-RAD T100 thermocycler with

35430 cycles of $30 \mathrm{~s}$ at $95^{\circ} \mathrm{C}, 30 \mathrm{~s}$ at $66.7^{\circ} \mathrm{C}$ and $60 \mathrm{~s}$ at $72^{\circ} \mathrm{C}$.

355 T7 endonuclease assay. PCR products containing GLTSCR1L-induced in-dels were purified by

356 MN PCR-Clean up Kit. 400 ng of purified DNA was denatured and heteroduplexed using

357 NEBuffer 2 (catalog no. B7002S) in a total volume of $19 \mu \mathrm{L}$ with the following settings in a

358 thermocycler: 5 minutes at $95^{\circ} \mathrm{C}$, ramping down to $85^{\circ} \mathrm{C}$ with $2{ }^{\circ} \mathrm{C} / \mathrm{s}$, ramping down to $25^{\circ} \mathrm{C}$

359 with $0.1{ }^{\circ} \mathrm{C} / \mathrm{s} .1 \mu \mathrm{L} \mathrm{T} 7$ endonuclease (catalog no. M0302L) was added into the heteroduplexed

360 DNA reaction and mixed thoroughly. The reaction was incubated at $37{ }^{\circ} \mathrm{C}$ for 1 hour and

361 visualized in BIO-RAD Gel Doc XR+ after running on agarose gels. 
363 Flow cytometry. Cell surface TRA-1-60 expression was analyzed with an Accuri C6 flow

364 cytometer (BD) using PE-conjugated anti-human TRA-1-60-R antibody (Biolegend, catalog no.

$365330610)$

366 Quantitative RT-PCR analyses. qPCR assays were performed as described previously (10)

367 with the indicated primers (Table S1).

368 Cell proliferation assay. OCT4-GFP iPSCs were seeded on matrigel-coated 96-well plates in

369 mTESR1 media with ROCK inhibitor Y-27632 $(10 \mu \mathrm{M})$. When cells reach $40-60 \%$ confluency,

370 they were treated with DMSO, $1 \mu \mathrm{M}$ I-BRD9 or $0.1 \mu \mathrm{M}$ dBRD9 in mTESR1 media for 48 hours.

371 Cell proliferation was measured with the CellTiter-Glo Luminescent Cell Viability Assay

372 (Promega) according to manufacturer's instructions using luminometric measurements with a

373 plate reader (Synergy H1 Reader, BioTek).

374 Immunostaining. TRA-1-60 staining was performed as previously described (4) and anti-

375 SSEA1 antibody (Biolegend, catalog no. 125604) was used to quantify MEF reprogramming.

376 For immunofluorescence-based characterization of iPSCs, cells were passaged onto mitomycin-

377 C-treated MEFs in hESC medium and carried out as previously described (10). The antibodies

378 used were OCT4 (Abcam, catalog no. ab19857), SSEA4/A647 (BD, catalog no. 560218) and

379 NANOG (Abcam, catalog no. ab21624). For NANOG and OCT4, Alexa-488-conjugated

380 secondary antibodies (Molecular Probes) were used. Nuclei were stained with VECTASHIELD

381 Antifade mounting medium with DAPI (H-1200-10).

382 Teratoma assays. All experiments were carried out under a protocol approved by Koç

383 University Animal Experiments Ethics Committee and all relevant ethical regulations were

384 complied with. Teratoma injections were performed as previously described (53). 
RNA-sequencing and analysis. Fibroblasts were treated for 5 days with DMSO, BI-7273 (1

samples. Genes were considered as differentially regulated based on adjusted $p$-value $<0.05$. The

394 formation of fibroblast-related gene set was described previously (10). Rank-ordered gene lists

395 were used for gene-set enrichment analysis (54).

396 ATAC-Sequencing and analysis. ATAC-seq was performed using 100,000 cells for the

397 transposition reaction as described previously (55) using in-house-produced Tn5 transposase.

398 Subsequently, samples were purified using the GeneJET PCR purification kit (Thermo). PCR

399 amplification was performed using the following protocol: $3 \mathrm{~min}$ at $72{ }^{\circ} \mathrm{C}, 30 \mathrm{~s}$ at $98^{\circ} \mathrm{C}$ and 11

400 cycles of $10 \mathrm{~s}$ at $98^{\circ} \mathrm{C}, 30 \mathrm{~s}$ at $63^{\circ} \mathrm{C}$, and $3 \mathrm{~min}$ at $72^{\circ} \mathrm{C}$. The samples were then purified using

401 the GeneJET PCR purification kit and eluted with $20 \mu \mathrm{l}$ of TE buffer. Samples were then run on

402 a Tapestation (Agilent) to determine library size and quantification before paired-end $(2 \times 41 \mathrm{bp})$

403 sequencing on a NextSeq 500 (Illumina) platform. Sequence reads were quality controlled with

404 FastQC and mapped to the most recent human reference genome (hg38) using bowtie (56),

405 allowing a maximum of 2 mismatches and only uniquely mapped reads. Reads with mapping

406 quality score below 30 and blacklisted regions were filtered. DeepTools (57) ComputeMatrix

407 and plotProfile commands were used to generate aggregate ATAC plots. For this, BigWig files

408 were generated using deepTools bamCoverage command, eliminating duplicates and 
normalizing by sequencing depth and effective genome size. Intersecting regions among

410 fibroblast ChIP peaks for H3K27ac, H3K4me1 and H3K4me3 were identified using previously

411 published fibroblast ChIP-seq data (10), calling the peaks using macs2 (58) with the parameters -

412 -keep-dup 1 and -broad, and finding the intersecting regions using bedtools (59) intersect.

\section{Statistical Analysis}

414 Statistical analysis was performed using GraphPad Prism 8 for t-tests or R 4.0.2 for Wilcoxon-

415 signed rank test. The test details, number of biological replicates and exact p-values were

416 provided in related figure legends.

\section{$417 \quad$ References}

418 1. K. Takahashi, K. Tanabe, M. Ohnuki, M. Narita, T. Ichisaka, K. Tomoda, S. Yamanaka, 419 Induction of Pluripotent Stem Cells from Adult Human Fibroblasts by Defined Factors.

$420 \quad$ Cell. 131, 861-872 (2007).

$421 \quad$ 2. K. Takahashi, S. Yamanaka, Induction of Pluripotent Stem Cells from Mouse Embryonic 422 and Adult Fibroblast Cultures by Defined Factors. Cell. 126, 663-676 (2006).

423 3. B. Papp, K. Plath, Epigenetics of Reprogramming to Induced Pluripotency. Cell. 152, $424 \quad 1324-1343(2013)$.

425 4. T. T. Onder, N. Kara, A. Cherry, A. U. Sinha, N. Zhu, K. M. Bernt, P. Cahan, B. O.

426 Marcarci, J. Unternaehrer, P. B. Gupta, E. S. Lander, S. A. Armstrong, G. Q. Daley, 427 Chromatin-modifying enzymes as modulators of reprogramming. Nature. 483, 598-602 $428 \quad$ (2012).

429 5. S. Cheloufi, U. Elling, B. Hopfgartner, Y. L. Jung, J. Murn, M. Ninova, M. Hubmann, A. 430 I. Badeaux, C. Euong Ang, D. Tenen, D. J. Wesche, N. Abazova, M. Hogue, N. Tasdemir, 431 J. Brumbaugh, P. Rathert, J. Jude, F. Ferrari, A. Blanco, M. Fellner, D. Wenzel, M. 
Zinner, S. E. Vidal, O. Bell, M. Stadtfeld, H. Y. Chang, G. Almouzni, S. W. Lowe, J.

528, 218-224 (2015).

6. Z. Shao, C. Yao, A. Khodadadi-Jamayran, W. Xu, T. M. Townes, M. R. Crowley, K. Hu,

$4397 . \quad$ C. J. Lynch, R. Bernad, I. Calvo, S. Nóbrega-Pereira, S. Ruiz, N. Ibarz, A. Martinez-Val,

444 8. J.-C. Cossec, I. Theurillat, C. Chica, S. Búa Aguín, X. Gaume, A. Andrieux, A. Iturbide,

9. E. Kolundzic, A. Ofenbauer, S. I. Bulut, B. Uyar, G. Baytek, A. Sommermeier, S. Seelk, Cells. Dev. Cell. 46, 611-626.e12 (2018). 
Biol. 15, 519-528 (2019).

11. J. Brumbaugh, B. Di Stefano, K. Hochedlinger, Reprogramming: identifying the mechanisms that safeguard cell identity. Development. 146, dev182170 (2019).

12. S. K. Hota, B. G. Bruneau, ATP-dependent chromatin remodeling during mammalian development. Development. 143, 2882-2897 (2016).

13. C. Hodges, J. G. Kirkland, G. R. Crabtree, The Many Roles of BAF (mSWI/SNF) and PBAF Complexes in Cancer. Cold Spring Harb. Perspect. Med. . 6 (2016), doi:10.1101/cshperspect.a026930.

14. C. Kadoch, R. T. Williams, J. P. Calarco, E. L. Miller, C. M. Weber, S. M. G. Braun, J. L. Pulice, E. J. Chory, G. R. Crabtree, Dynamics of BAF-Polycomb complex opposition on pluripotency by conditioning the genome for LIF/STAT3 signalling and by regulating

16. R. L. dos Santos, L. Tosti, A. Radzisheuskaya, I. M. Caballero, K. Kaji, B. Hendrich, J. C. Manner. Cell Stem Cell. 15, 102-110 (2014).

472 17. Q. Zhuang, W. Li, C. Benda, Z. Huang, T. Ahmed, P. Liu, X. Guo, D. P. Ibañez, Z. Luo, 
478 18. N. Singhal, J. Graumann, G. Wu, M. J. Araúzo-Bravo, D. W. Han, B. Greber, L. Gentile,

19. L. Wang, Y. Du, J. M. Ward, T. Shimbo, B. Lackford, X. Zheng, Y. Miao, B. Zhou, L. and Blastocyst Development. Cell Stem Cell. 14, 575-591 (2014).

20. Z. Jiang, Y. Tang, X. Zhao, M. Zhang, D. M. Donovan, X. (Cindy) Tian, Knockdown of Brm and Baf170, Components of Chromatin Remodeling Complex, Facilitates Reprogramming of Somatic Cells. Stem Cells Dev. 24, 2328-2336 (2015).

488 21. A. Alpsoy, E. C. Dykhuizen, Glioma tumor suppressor candidate region gene 1 (GLTSCR1) and its paralog GLTSCR1-like form SWI/SNF chromatin remodeling subcomplexes. J. Biol. Chem. 293, 3892-3903 (2018).

491 22. N. Mashtalir, A. R. D’Avino, B. C. Michel, J. Luo, J. Pan, J. E. Otto, H. J. Zullow, Z. M. McKenzie, R. L. Kubiak, R. St Pierre, A. M. Valencia, S. J. Poynter, S. H. Cassel, J. A. Ranish, C. Kadoch, Modular Organization and Assembly of SWI/SNF Family Chromatin Remodeling Complexes. Cell. 175, 1272-1288 (2018).

495 23. B. C. Michel, A. R. D’Avino, S. H. Cassel, N. Mashtalir, Z. M. McKenzie, M. J. McBride, A. M. Valencia, Q. Zhou, M. Bocker, L. M. M. Soares, J. Pan, D. I. Remillard, C. A. non-canonical SWI/SNF complex is a synthetic lethal target in cancers driven by BAF

500 24. X. Wang, S. Wang, E. C. Troisi, T. P. Howard, J. R. Haswell, B. K. Wolf, W. H. Hawk, P. 
Ramos, E. M. Oberlick, E. P. Tzvetkov, A. Ross, F. Vazquez, W. C. Hahn, P. J. Park, C.

W. M. Roberts, BRD9 defines a SWI/SNF sub-complex and constitutes a specific

vulnerability in malignant rhabdoid tumors. Nat. Commun. 10, 1881 (2019).

504 25. J. Pan, R. M. Meyers, B. C. Michel, N. Mashtalir, A. E. Sizemore, J. N. Wells, S. H.

26. N. Del Gaudio, A. Di Costanzo, N. Q. Liu, L. Conte, A. Migliaccio, M. Vermeulen, J. H.

10, 338 (2019).

512 27. J. Gatchalian, S. Malik, J. Ho, D.-S. Lee, T. W. R. Kelso, M. N. Shokhirev, J. R. Dixon,

28. P. G. K. Clark, L. C. C. Vieira, C. Tallant, O. Fedorov, D. C. Singleton, C. M. Rogers, O. 
Active Selective BRD9 Inhibitor. J. Med. Chem. 59, 4462-4475 (2016).

30. N. H. Theodoulou, P. Bamborough, A. J. Bannister, I. Becher, R. A. Bit, K. H. Che, C. W.

Chung, A. Dittmann, G. Drewes, D. H. Drewry, L. Gordon, P. Grandi, M. Leveridge, M.

K. Prinjha, P. G. Humphreys, Discovery of I-BRD9, a Selective Cell Active Chemical

Probe for Bromodomain Containing Protein 9 Inhibition. J. Med. Chem. 59, 1425-1439 (2016).

531 31. D. Remillard, D. L. Buckley, J. Paulk, G. L. Brien, M. Sonnett, H. S. Seo, S. Dastjerdi, M. Wühr, S. Dhe-Paganon, S. A. Armstrong, J. E. Bradner, Degradation of the BAF Complex Factor BRD9 by Heterobifunctional Ligands. Angew. Chemie - Int. Ed. 56, 5738-5743 (2017).

32. A. Q. Lam, B. S. Freedman, R. Morizane, P. H. Lerou, M. T. Valerius, J. V Bonventre, Rapid and efficient differentiation of human pluripotent stem cells into intermediate mesoderm that forms tubules expressing kidney proximal tubular markers. J. Am. Soc. Nephrol. 25, 1211-1225 (2014).

33. D. Balboa, J. Weltner, Y. Novik, S. Eurola, K. Wartiovaara, T. Otonkoski, Generation of an OCT4 reporter human induced pluripotent stem cell line using CRISPR/SpCas9. Stem

542 34. D. Li, J. Liu, X. Yang, C. Zhou, J. Guo, C. Wu, Y. Qin, L. Guo, J. He, S. Yu, H. Liu, X. Dynamics during iPSC Reprogramming. Cell Stem Cell. 21, 819-833.e6 (2017). 
D. Horn, C. Troyer, S. G. Kant, Y. Lee, G. E. Ishak, G. Leung, A. Barone Pritchard, S.

Waggoner, K. W. Gripp, M. J. Parker, J. Stoler, S. Lyonnet, V. Cormier-Daire, D. rhombencephalosynapsis. Brain. 143, 55-68 (2020).

36. M. A. Meester-Smoor, M. J. F. W. Janssen, G. C. Grosveld, A. de Klein, W. F. J. van

IJcken, H. Douben, E. C. Zwarthoff, MN1 affects expression of genes involved in hematopoiesis and can enhance as well as inhibit RAR/RXR-induced gene expression.

564 37. S. S. Riedel, J. N. Haladyna, M. Bezzant, B. Stevens, D. A. Pollyea, A. U. Sinha, S. A. Armstrong, Q. Wei, R. M. Pollock, S. R. Daigle, C. T. Jordan, P. Ernst, T. Neff, K. M. leukemia. J. Clin. Invest. 126, 1438-1450 (2016).

38. D. HniszBrian, D. Hnisz, B. J. Abraham, T. I. Lee, A. Lau, V. Saint-André, A. A. Sigova, H. A. Hoke, R. A. Young, D. HniszBrian, Super-Enhancers in the Control of Cell Identity 
and Disease. Cell. 155, 934-947 (2013).

39. L. Ho, J. L. Ronan, J. Wu, B. T. Staahl, L. Chen, A. Kuo, J. Lessard, A. I. Nesvizhskii, J.

Ranish, G. R. Crabtree, An embryonic stem cell chromatin remodeling complex, esBAF, is essential for embryonic stem cell self-renewal and pluripotency. Proc. Natl. Acad. Sci. U. S. A. 106, 5181-6 (2009).

40. K. Fu, C. Chronis, A. Soufi, G. Bonora, M. Edwards, S. T. Smale, K. S. Zaret, K. Plath, M. Pellegrini, Comparison of reprogramming factor targets reveals both species-specific and conserved mechanisms in early iPSC reprogramming. BMC Genomics. 19, 956 (2018).

41. K.-P. Kim, Y. Wu, J. Yoon, K. Adachi, G. Wu, S. Velychko, C. M. MacCarthy, B. Shin, domains. Sci. Adv. 6 (2020), doi:10.1126/sciadv.aaz7364.

42. A. Janiszewski, I. Talon, J. Chappell, S. Collombet, J. Song, N. De Geest, S. K. To, G. reprogramming. Genome Res. 29, 1659-1672 (2019).

587 43. M. C. Bell, P. Raffeiner, R. J. Hart, K. P. Vogt, PIK3CA Cooperates with KRAS to 11 (2019), , doi:10.3390/cancers11111634. 
Bradley, Spliceosomal disruption of the non-canonical BAF complex in cancer. Nature. 574, 432-436 (2019).

45. C.-S. Loo, J. Gatchalian, Y. Liang, M. Leblanc, M. Xie, J. Ho, B. Venkatraghavan, D. C. Hargreaves, Y. Zheng, A Genome-wide CRISPR Screen Reveals a Role for the Noncanonical Nucleosome-Remodeling BAF Complex in Foxp3 Expression and Regulatory T Cell Function. Immunity. 53, 143-157.e8 (2020).

46. I. Mellis, H. Edelstein, R. Truitt, L. Beck, O. Symmons, Y. Goyal, M. Dunagin, R. doi:10.1101/2020.06.11.147207.

47. Y. Zhao, T. Zhao, J. Guan, X. Zhang, Y. Fu, J. Ye, J. Zhu, G. Meng, J. Ge, S. Yang, L. Cheng, Y. Du, C. Zhao, T. Wang, L. Su, W. Yang, H. Deng, A XEN-like State Bridges

48. K.-P. Kim, J. Choi, J. Yoon, J. M. Bruder, B. Shin, J. Kim, M. J. Arauzo-Bravo, D. Han, reprogramming competence to transcriptional regulators. Nat. Chem. Biol. 17, 47-56 (2021). defined factors. Nature. 451, 141-146 (2008).

613 50. K. Fidan, G. Kavaklığlu, A. Ebrahimi, C. Özlü, N. Z. Ay, A. Ruacan, A. Gül, T. T. Önder, Generation of integration-free induced pluripotent stem cells from a patient with 
616 51. C. A. Sommer, M. Stadtfeld, G. J. Murphy, K. Hochedlinger, D. N. Kotton, G. Mostoslavsky, Induced pluripotent stem cell generation using a single lentiviral stem cell cassette. Stem Cells. 27, 543-549 (2009).

619 52. A. Alici-Garipcan, B. Özçimen, I. Süder, V. Ülker, T. T. Önder, N. Özören, NLRP7 plays a functional role in regulating BMP4 signaling during differentiation of patient-derived trophoblasts. Cell Death Dis. 11, 658 (2020).

53. K. Fidan, A. Ebrahimi, Ö. H. Çağlayan, B. Özçimen, T. T. Önder, in Methods in molecular biology (Clifton, N.J.) (2015), vol. 1353, pp. 215-231.

54. A. Subramanian, P. Tamayo, V. K. Mootha, S. Mukherjee, B. L. Ebert, M. A. Gillette, A. analysis: a knowledge-based approach for interpreting genome-wide expression profiles.

55. J. D. Buenrostro, P. G. Giresi, L. C. Zaba, H. Y. Chang, W. J. Greenleaf, Transposition of native chromatin for fast and sensitive epigenomic profiling of open chromatin, DNA-

56. B. Langmead, C. Trapnell, M. Pop, S. L. Salzberg, Ultrafast and memory-efficient alignment of short DNA sequences to the human genome. Genome Biol. 10, R25 (2009).

633 57. F. Ramírez, D. P. Ryan, B. Grüning, V. Bhardwaj, F. Kilpert, A. S. Richter, S. Heyne, F. Dündar, T. Manke, deepTools2: a next generation web server for deep-sequencing data analysis. Nucleic Acids Res. 44, W160-5 (2016). 
59. A. R. Quinlan, I. M. Hall, BEDTools: a flexible suite of utilities for comparing genomic features. Bioinformatics. 26, 841-842 (2010).

\section{Acknowledgments}

We would like to thank Ahmet Kocabay and Ali Cihan Taşkın for help with mouse

644 experiments, Arzu Ruacan (Koç University, School of Medicine, Department of Pathology) for

645 examination of histological sections. The authors gratefully acknowledge use of the services and

646 facilities of the Koç University Research Center for Translational Medicine (KUTTAM), funded

647 by the Republic of Turkey Ministry of Development. The content is solely the responsibility of

648 the authors and does not necessarily represent the official views of the Ministry of Development.

649 KS and DHA were supported by TUBITAK BIDEB Scholarship.

651 Funding:

652

Newton Advanced Fellowship (TTO)

TUBITAK Projects 231S182 and 219Z209 (TTO)

Arthritis Research UK, program grant 20522 (UO)

Cancer Research UK (UO)

656

LEAN project of the Leducq Foundation (UO)

661 Conceptualization: KS, GGS, UO, TTO 
662 Software: KS, ADC, APC

663 Formal analysis: KS, GGS, ADC, TTO

664 Investigation: KS, GGS, MP, SK, HC, ESA, HAD

665 Resources: APC, JED, ABD, LHS, JQ

666 Data Curation: KS, ADC, MP, APC

667 Writing — original draft: KS, TTO

668 Writing — review \& editing: GGS, ADC, SK, JQ, UO

669 Supervision: JQ, UO, TTO

670 Funding acquisition: UO, TTO

671

672 Competing interests: Authors declare that they have no competing interests.

673

674 Data and materials availability:

675 RNA-sequencing and ATAC-sequencing data are deposited to the NCBI GEO database with the

676 accession number GSE161640. Necessary information to produce materials and replicate

677 analysis is provided in the main text or the supplementary materials.

678 


\section{Figure 1}
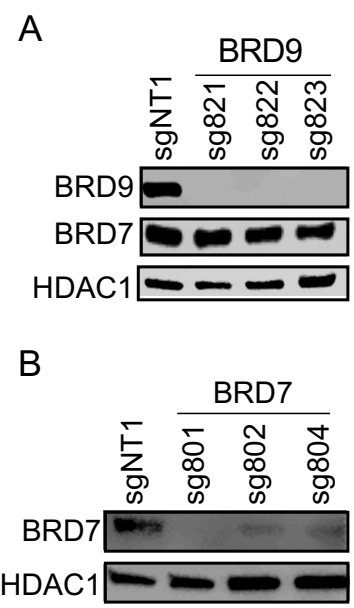

681

682

683

684

685

686

687

688

689

690

691

692

693

694

695

696

697

698

699

700

701
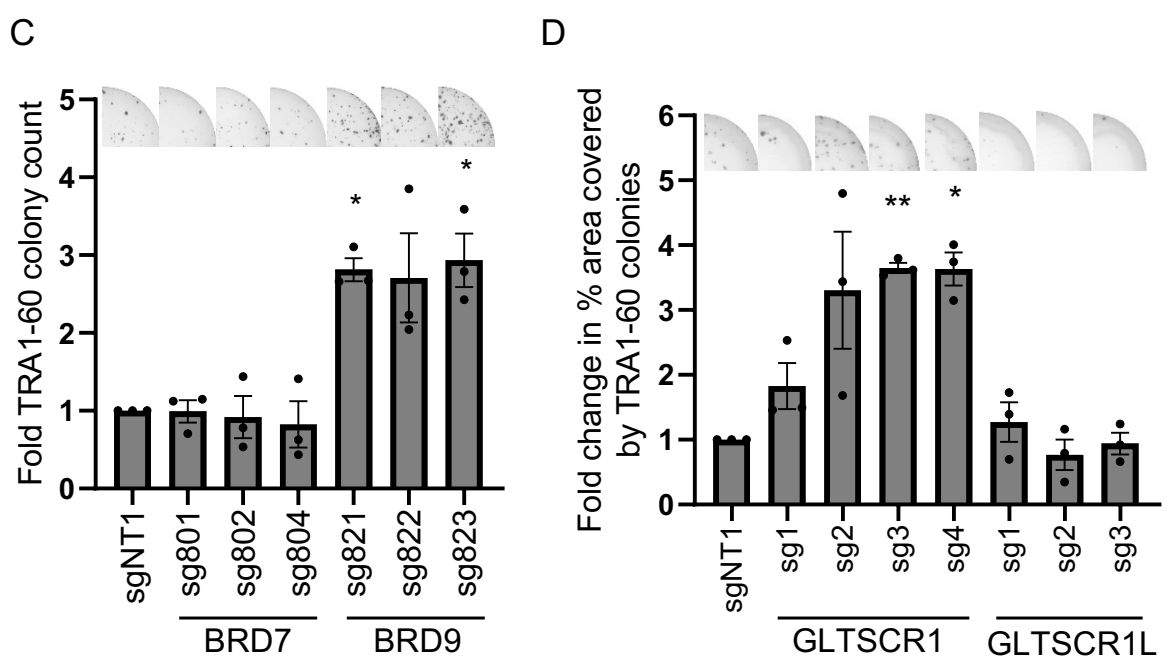

\section{Figure 1. Genetic suppression of ncBAF-specific subunits increases reprogramming} efficiency. (A) Western blots for BRD9 and BRD7 in fibroblasts expressing control (sgNT1) and BRD9-targeting gRNAs. HDAC1 serves as loading control. (B) Western blots for BRD7 in fibroblasts expressing control (sgNT1) and BRD7-targeting gRNAs. HDAC1 serves as loading control. (C) Fold change in the number of TRA-1-60-positive colonies for indicated sgRNAexpressing cells compared to control sgNT1-expressing cells. Representative well images are shown above the graph. Bar graphs show the mean and error bars represent standard error of mean. $\mathrm{N}=3$, biological replicates. P-values are calculated by one sample t-test for $\mathrm{mu}=1$. * denotes $\mathrm{p}<0.05$, exact $\mathrm{p}$-values from left to right are: $0.9593,0.7910,0.6173,0.0065,0.0968$, 0.0302. (D) Fold change in percent area covered by TRA-1-60-positive colonies generated from fibroblasts expressing GLTSCR1 or GLTSCR IL sgRNAs compared to control sgNT1.

Representative well images are above relative bars. Bar graphs show the mean and error bars represent standard error of mean. $\mathrm{N}=3$, three biological replicates. P-values are calculated by one sample t-test for $\mathrm{mu}=1$. $*$ denotes $\mathrm{p}<0.05, * *$ denotes $\mathrm{p}<0.005$, exact $\mathrm{p}$-values from left to right are: $0.1442,0.1248,0.0009,0.0092,0.4638,0.4252,0.7585$. 
Figure 2

A

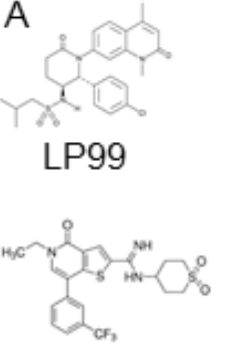

I-BRD9
Bl-7273

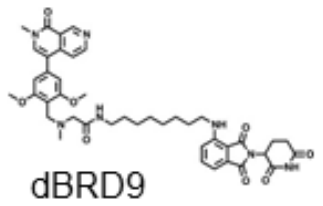

C

Time (h) $\quad 0 \quad 6 \quad 6 \quad 12 \quad 24 \quad 48 \quad 72$

BRD9

BRD7

HDAC1
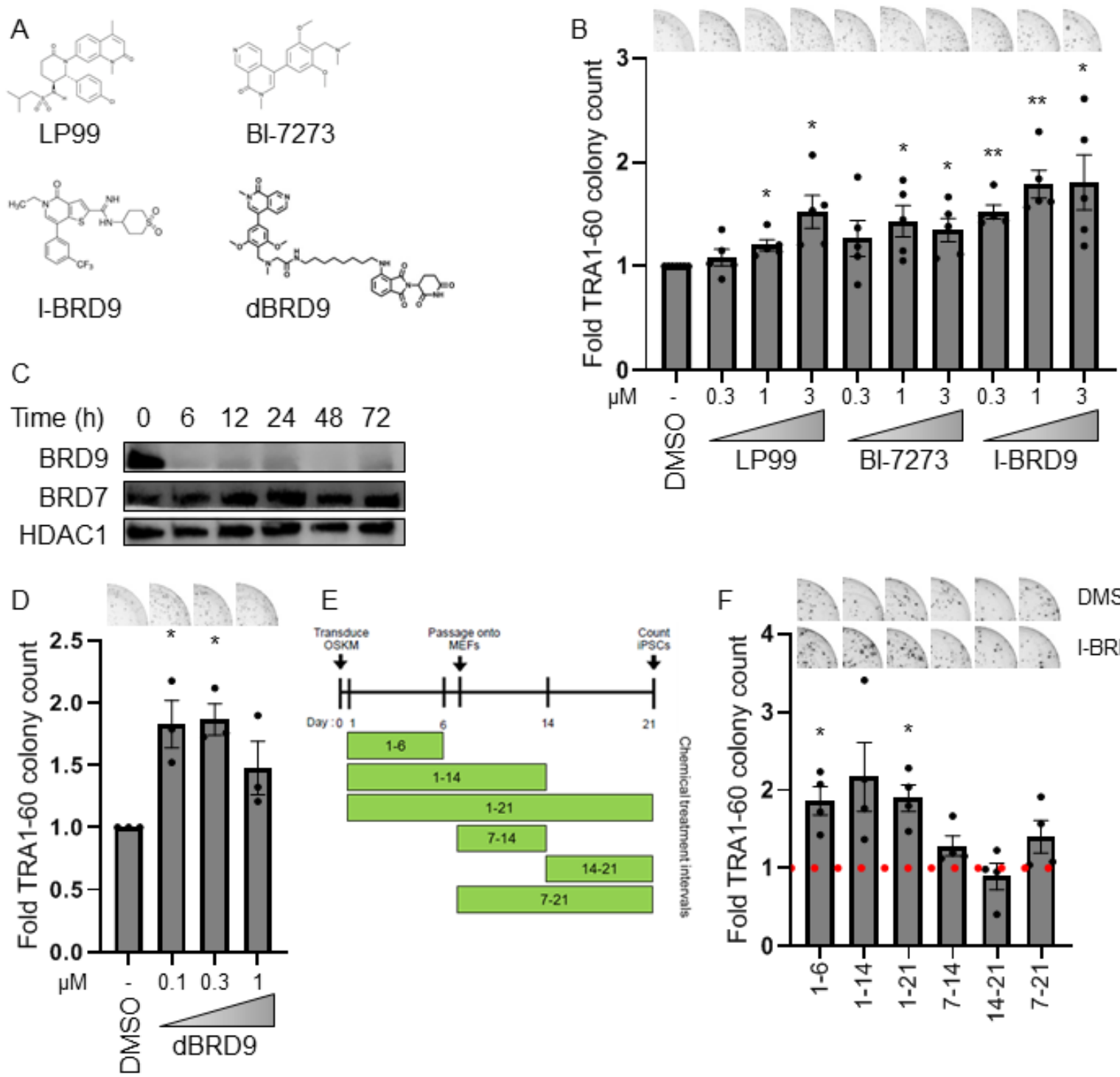

F

G
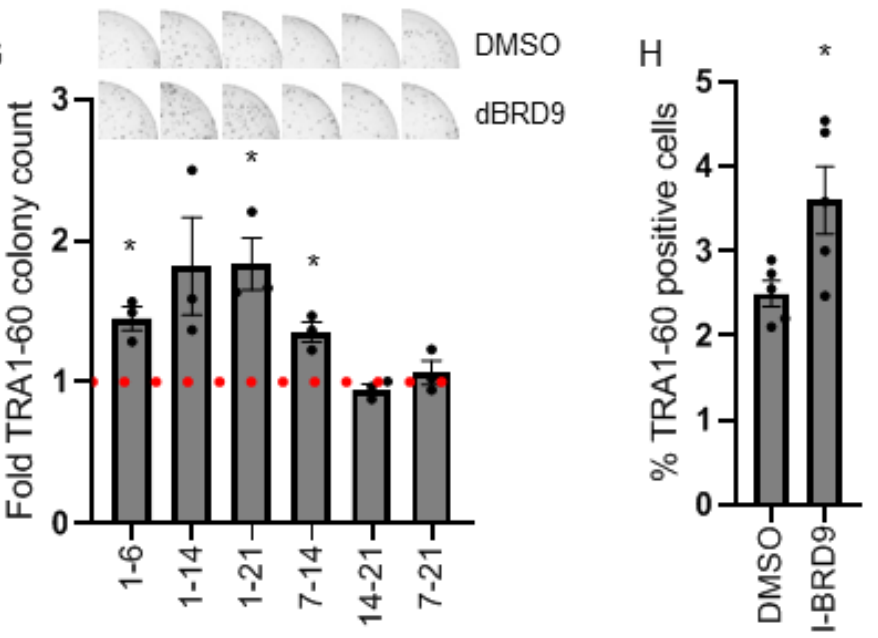
Figure 2. Bromodomain inhibition and degradation of BRD9 facilitate reprogramming. (A) Chemical structures of LP99, BI-7273, I-BRD9 and dBRD9. (B) Fold change in the number of TRA-1-60-positive colonies with the indicated compound treatments compared to DMSO control. Representative well images are shown above. Bar graphs show the mean and error bars represent standard error of mean. $n=5$, biological replicates for each treatment with 3 technical replicates. $\mathrm{p}$-values are calculated by one sample t-test for $\mathrm{mu}=1 .{ }^{*}$ denotes $\mathrm{p}<0.05, * *$ denotes $\mathrm{p}<0.005$, exact $\mathrm{p}$-values from left to right are: $0.3555,0.0175,0.0294,0.1954,0.0443,0.0364$, 0.0015, 0.0042, 0.0387. (C) Western blots for BRD9 and BRD7 at indicated time points after treatment with $0.3 \mu \mathrm{M}$ dBRD9. HDAC1 serves as loading control. (D) Fold change in the number of TRA-1-60-positive colonies with increasing concentrations of dBRD9 compared to DMSO. Representative well images are above the graph. Bar graphs show the mean and error bars represent standard error of mean. $n=3$, biological replicates. $p$-values are calculated by one sample t-test for $m u=1$. ${ }^{*}$ denotes $\mathrm{p}<0.05$, exact $\mathrm{p}$-values from left to right are: $0.0488,0.0204$, 0.1550. (E) Schematic depicting the time intervals for treatment of compounds during reprogramming. (F) Fold change in the number of TRA-1-60-positive colonies upon I-BRD9 treatment compared to DMSO between indicated days of reprogramming. Representative well images are above the graph. Bar graphs show the mean and error bars represent standard error of mean. $n=4$, independent biological replicates. $\mathrm{p}$-values were calculated by one sample t-test for $\mathrm{mu}=1 .{ }^{*}$ denotes $\mathrm{p}<0.05$, exact $\mathrm{p}$-values from left to right are: $0.0178,0.0771,0.0139,0.1116$, $0.5826,0.1492$. (G) Fold change in the number of TRA-1-60-positive colonies upon dBRD9 treatment compared to DMSO between indicated days of reprogramming. Representative well images are above the graph. Bar graphs show the mean and error bars represent standard error of mean. $n=3$, independent biological replicates. $p$-values are calculated by one sample $\mathrm{t}$-test for $\mathrm{mu}=1 . *$ denotes $\mathrm{p}<0.05$, exact $\mathrm{p}$-values from left to right are: $0.0332,0.1417,0.0456,0.0376$, 0.3099, 0.5208. (H) Percentage of TRA-1-60-positive cells on day 6 of reprogramming with of mean. $\mathrm{n}=5$, five biological replicates. $\mathrm{p}$-values are calculated by two sample t-test. * denotes $\mathrm{p}<0.05$, exact $\mathrm{p}$-value: 0.0474 . 


\section{Figure 3}
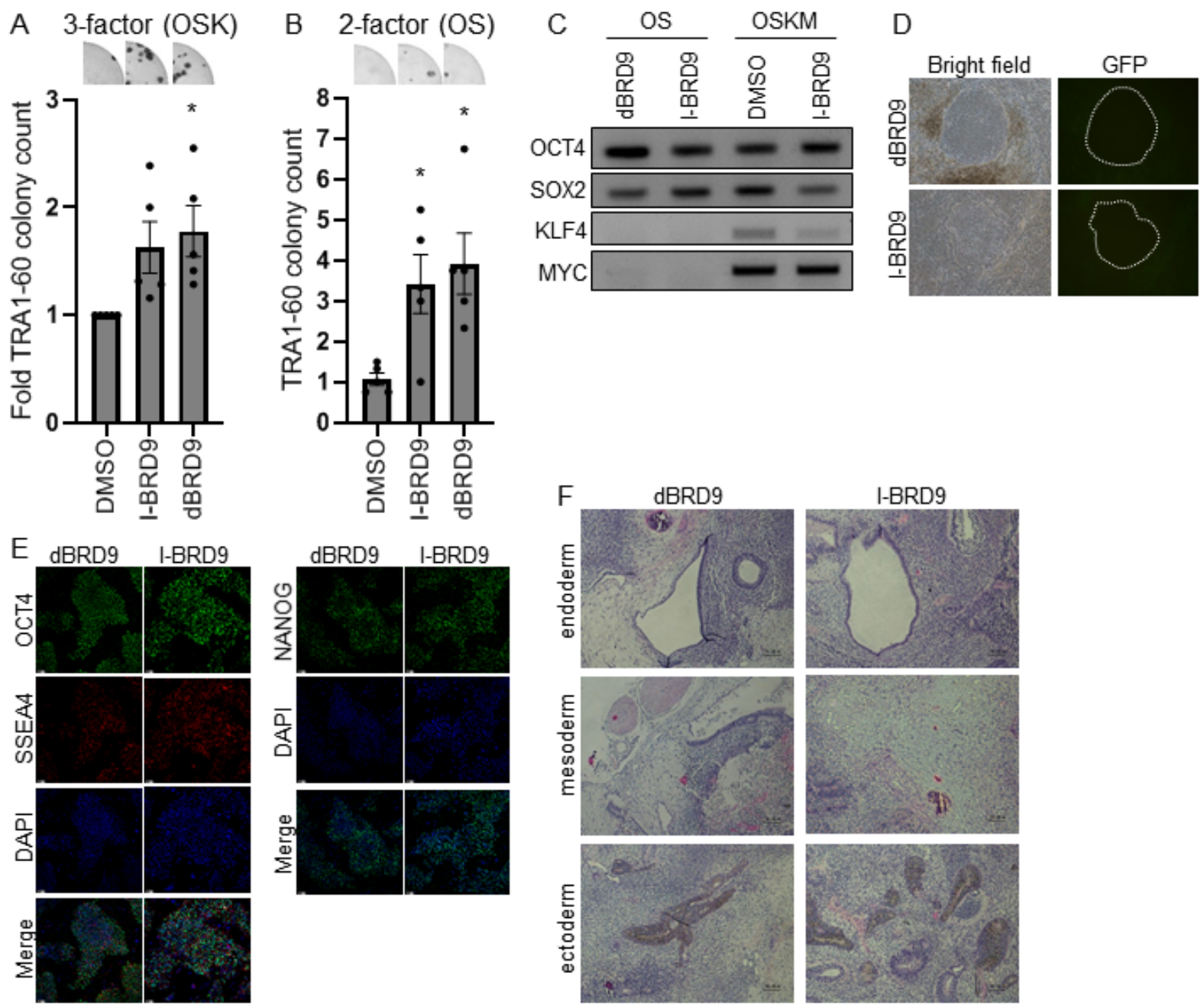

Figure 3. BRD9 inhibition and degradation enable iPSC generation without KLF4 and cMYC. (A) Fold change in the number of TRA-1-60-positive colonies generated by OCT4, SOX2 and KLF4 (OSK) in the presence of DMSO, I-BRD9 or dBRD9. Representative well images are above the graph. Bar graphs show the mean and error bars represent standard error of mean. $\mathrm{n}=5$, five biological replicates. $p$-values are calculated by one sample t-test for $m u=1$. Exact $p$-values from left to right are: $0.0583,0.0297$. (B) Number of TRA-1-60 positive colonies generated by OCT4 and SOX2 (OS) in the presence of DMSO, I-BRD9 or dBRD9. Representative well images are above the graph. $n=5$, biological replicates with at least 3 technical replicates. $p$ values are calculated by two sample t-test. ${ }^{*}$ denotes $\mathrm{p}<0.05$, exact $\mathrm{p}$-values from left to right are: 0.0302, 0.0182. (C) Agarose gel images of PCR products for exogenous OCT4, SOX2, KLF4 and c-MYC transgenes in iPSCs generated from fibroblasts with indicated induction and treatments. (D) Phase contrast and GFP fluorescence images of colonies derived by OS induction and dBRD9 (upper) or I-BRD9 (lower) treatments showing typical iPSC morphology and silencing of retroviral GFP transgene. (E) OCT4, SSEA4 (left) and NANOG (right) immunofluorescence of iPSCs derived from OS expressing fibroblasts treated with indicated treatments. Hoechst 33324 was used to stain the nuclei. (F) Hematoxylin and eosin stained 
bioRxiv preprint doi: https://doi.org/10.1101/2021.05.27.445940; this version posted May 29, 2021. The copyright holder for this preprint (which

was not certified by peer review) is the author/funder, who has granted bioRxiv a license to display the preprint in perpetuity. It is made available under aCC-BY-NC-ND 4.0 International license.

752 sections of teratomas of iPSCs derived from OS-expressing fibroblasts treated with dBRD9 or I753 BRD9 show tissues from endoderm, ectoderm and mesoderm lineages.

754 
bioRxiv preprint doi: https://doi.org/10.1101/2021.05.27.445940; this version posted May 29, 2021. The copyright holder for this preprint (which

was not certified by peer review) is the author/funder, who has granted bioRxiv a license to display the preprint in perpetuity. It is made available under aCC-BY-NC-ND 4.0 International license.

\section{Figure 4}

A

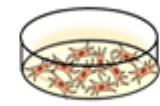

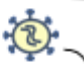

Cas9+

sgNT1
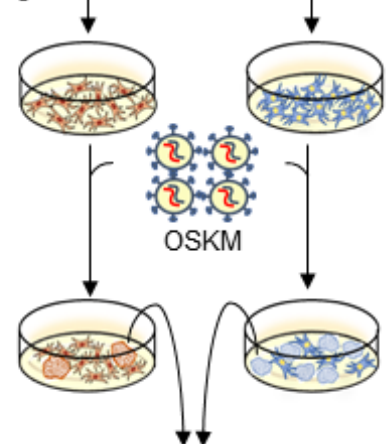

Pick and expand individual colonies

C

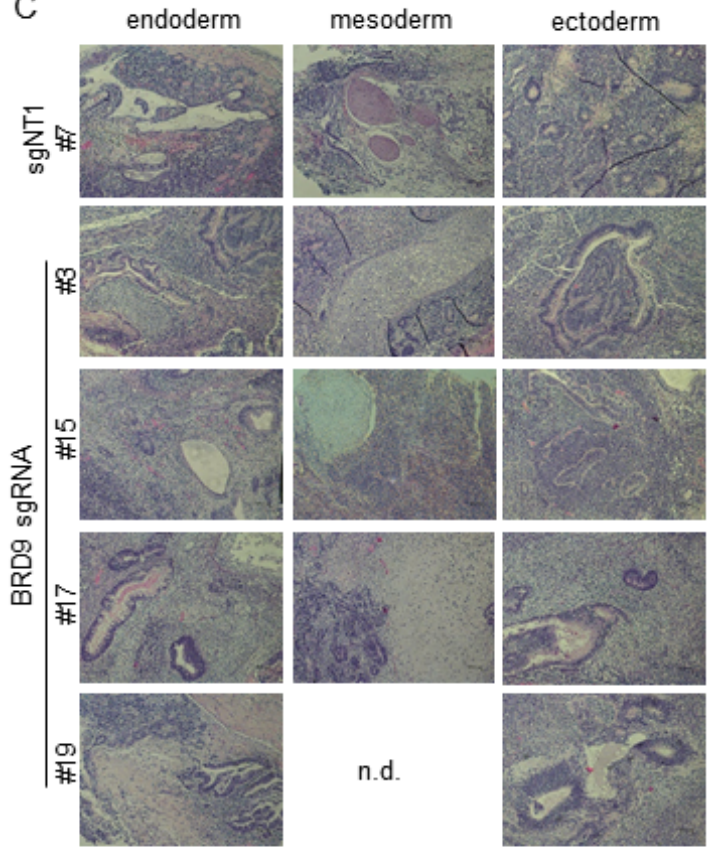

n.d.

BRD9 sgRNA

HDAC1

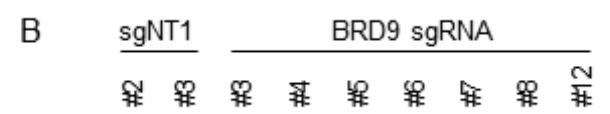

BRD9

HDAC1 -

$$
\text { sgNT1 BRD9 sgRNA }
$$
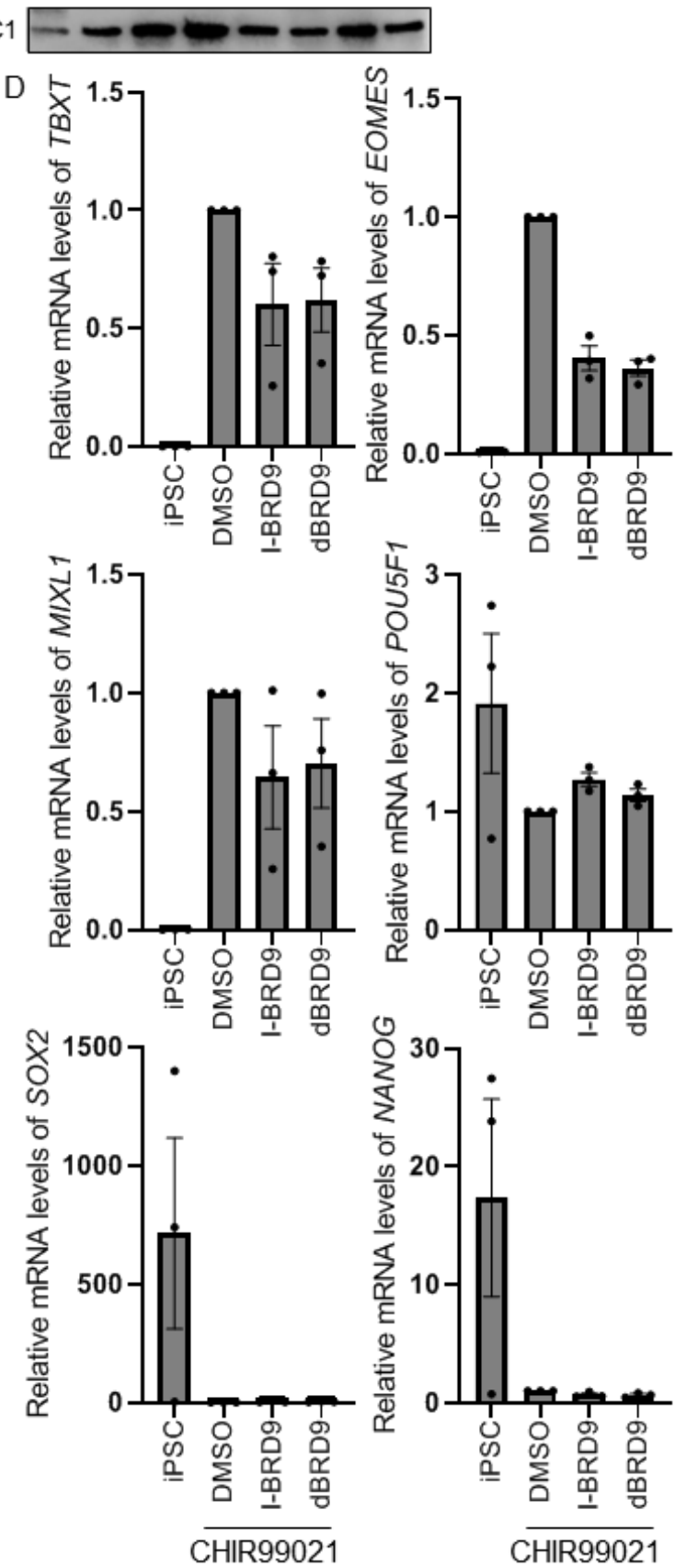
757 Figure 4. BRD9 is dispensable for human pluripotency induction and maintenance but 758 required for mesendedorm differentiation. (A) Schematic for generation of BRD9 knockout 759 and control iPSC lines (B) Western blot for BRD9 from iPSC clones generated from either

760 sgNT1 or BRD9 sg823-expressing fibroblasts. HDAC1 serves as loading control. (C)

761 Hematoxylin and eosin-stained sections of teratomas of iPSCs derived from sgNT1- or BRD9 762 sg823-expressing fibroblasts show tissues from endoderm, ectoderm and mesoderm lineages.

763 n.d.: not detected. (D) RT-qPCR results for mRNA levels of TBXT, EOMES, MIXL1, POU5F1, $764 S O X 2$ and $N A N O G$ genes normalized to $A C T B$ mRNA levels for indicated treatments. $\mathrm{n}=3$,

765 biological replicates. p-values are calculated by one sample t-test for $\mathrm{mu}=1$. Exact p-values of I766 BRD9 and dBRD9 treatments for TBXT expression are 0.1474 and 0.1069 , respectively. Exact p767 values of I-BRD9 and dBRD9 treatments for EOMES expression are 0.0076 and 0.0029,

768 respectively. Exact p-values of I-BRD9 and dBRD9 treatments for MIXL1 expression are 0.2429

769 and 0.2541 , respectively. Exact p-values of I-BRD9 and dBRD9 treatments for POU5F1

770 expression are 0.0436 and 0.1172 , respectively. Exact p-values of I-BRD9 and dBRD9

771 treatments for $S O X 2$ expression are 0.2104 and 0.0590 , respectively. Exact p-values of I-BRD9

772 and dBRD9 treatments for $N A N O G$ expression are 0.1376 and 0.0852 , respectively. 
bioRxiv preprint doi: https://doi.org/10.1101/2021.05.27.445940; this version posted May 29, 2021. The copyright holder for this preprint (which was not certified by peer review) is the author/funder, who has granted bioRxiv a license to display the preprint in perpetuity. It is made available under aCC-BY-NC-ND 4.0 International license.

\section{$774 \quad$ Figure 5}

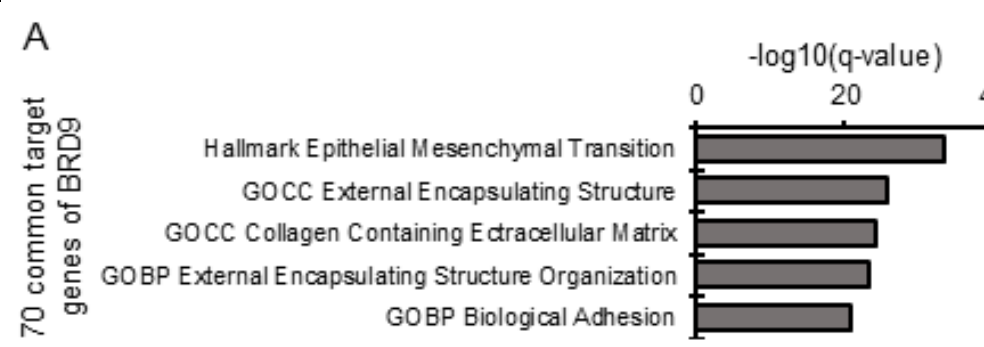

C

BI-7273 vs DMSO

Enrichment plot: FIBRO-RELATED GENE SET

NES: -2.38

q val: 0

要。

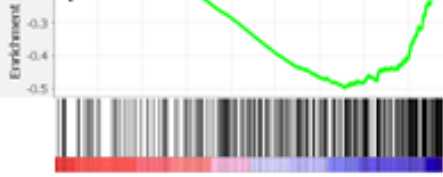

dBRD9 vs DMSO

Enrichment plot: FIBRO-RELATED GENE SET

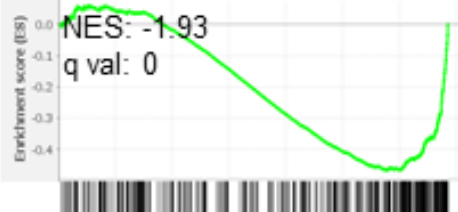

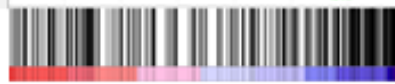

$\mathrm{D}$

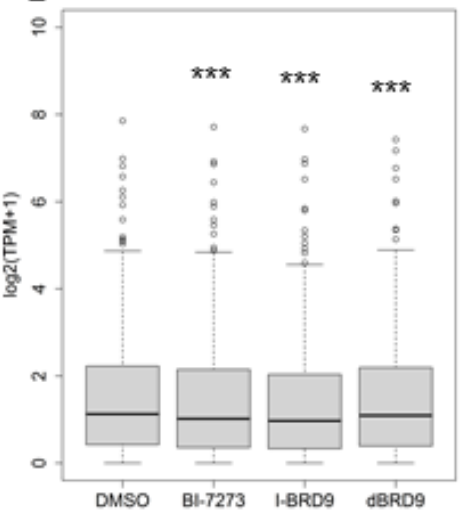

$\mathrm{F}$

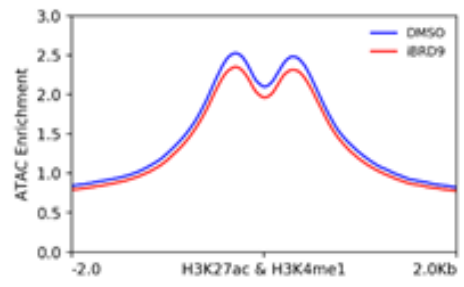

I-BRD9 vs DMSO

\section{Enrichment plot: FIBRO-RELATED GENE SET}

区.

q $q$ val: 0

है.0.5

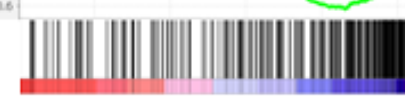

BRD9 sg823 vs sgNT1

Enrichment plot: FIBRO-RELATEO GENE SET
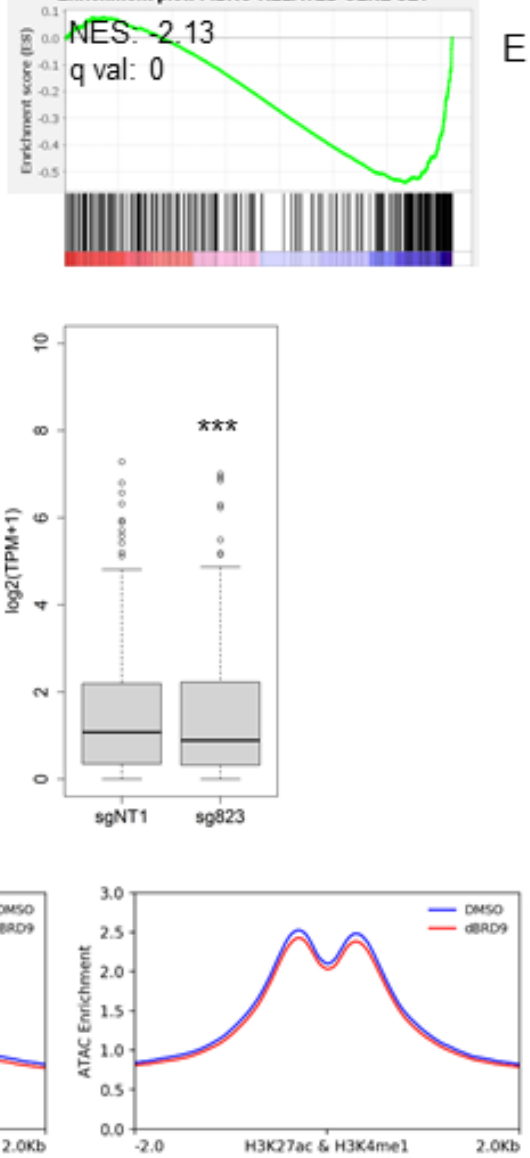

$E$
B I-BRD9 vs DMSO dBRD9 vs DMSO 40

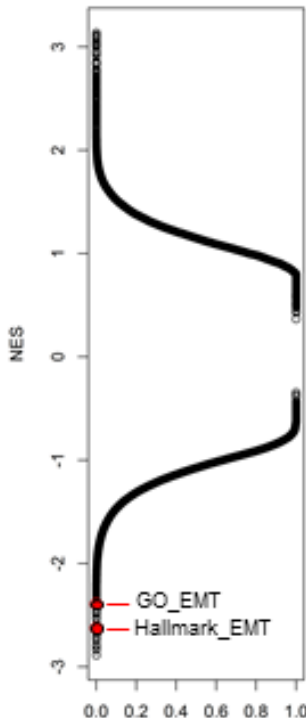

FDR q-value
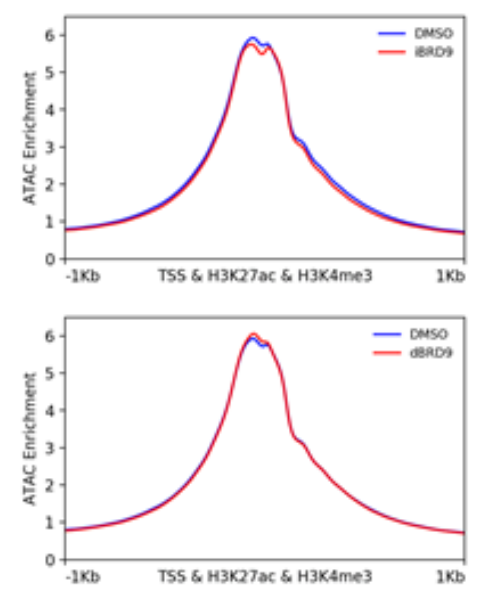
776 Figure 5. BRD9 maintains fibroblast-specific gene expression and enhancer accessibility.

777 (A) Top five GO and Hallmark gene sets enriched in common downregulated genes upon

778 dBRD9, BI-7273 and I-BRD9 treatments. Number of genes (n) in comparison were 70. p-values

779 were calculated by hypergeometric distribution. (B) Gene set enrichment analysis on pre-ranked

780 gene lists according to $\log 2 \mathrm{FC}$ value for comparisons of fibroblasts treated with I-BRD9 and

781 DMSO (left) and dBRD9 and DMSO (right) for all genesets available at The Molecular

782 Signatures Database (MSigDB). Red circles indicate Hallmark_EMT and GO_EMT gene sets.

783 (C) Gene set enrichment analysis (GSEA) of transcriptome data with indicated treatments for the

784 fibroblast-related gene set. NES: normalized enrichment score, q val: False discovery rate (FDR)

785 q-value. (D) Average expression levels of the 307 genes in fibroblast-related gene set across

786 indicated fibroblasts. Whiskers indicate 95\% confidence interval. p-values were calculated by

787 Wilcoxon signed-rank test. $* * *$ denotes $\mathrm{p}<0.005$, exact $\mathrm{p}$-values from left to right are: $2.2 \mathrm{e}-16$,

788 2.2e-16, 3.8e-10 and 5.057e-05. (E) Aggregate ATAC-seq plots from fibroblasts treated with I-

789 BRD9 (top), dBRD9 (bottom) and DMSO on the $+-1 \mathrm{~kb}$ of transcription start site, H3K27ac and

790 H3K4me3 summit. n=3, three biological replicates. (F) Aggregate ATAC-seq plots from

791 fibroblasts treated with I-BRD9 (left), dBRD9 (right) and DMSO around +/-2kb of H3K27ac and

792 H3K4me1 summit. $n=3$, three biological replicates.

793 
bioRxiv preprint doi: https://doi.org/10.1101/2021.05.27.445940; this version posted May 29, 2021. The copyright holder for this preprint (which was not certified by peer review) is the author/funder, who has granted bioRxiv a license to display the preprint in perpetuity. It is made available under aCC-BY-NC-ND 4.0 International license.

$794 \quad$ Figure 6.

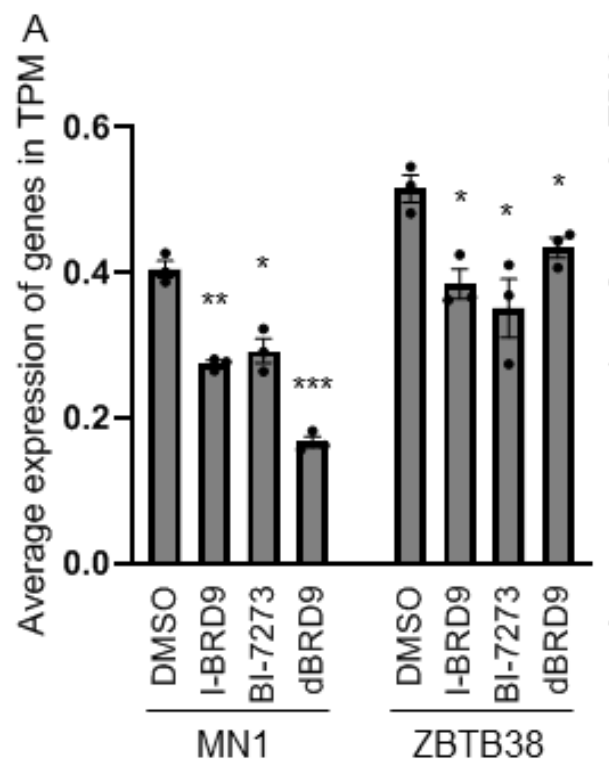

D

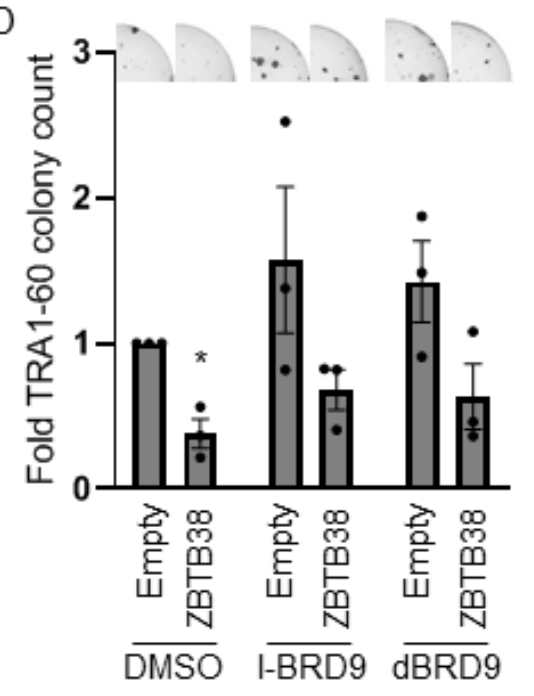

B
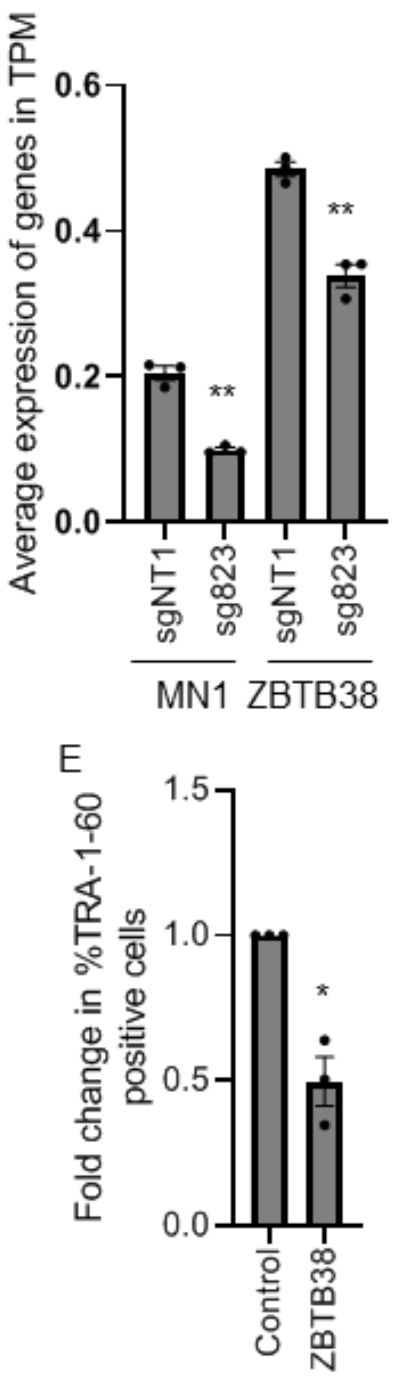

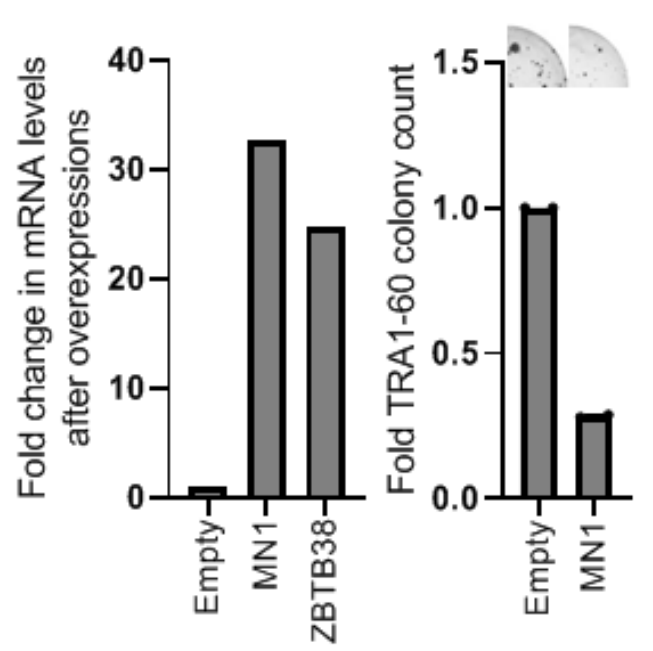

F

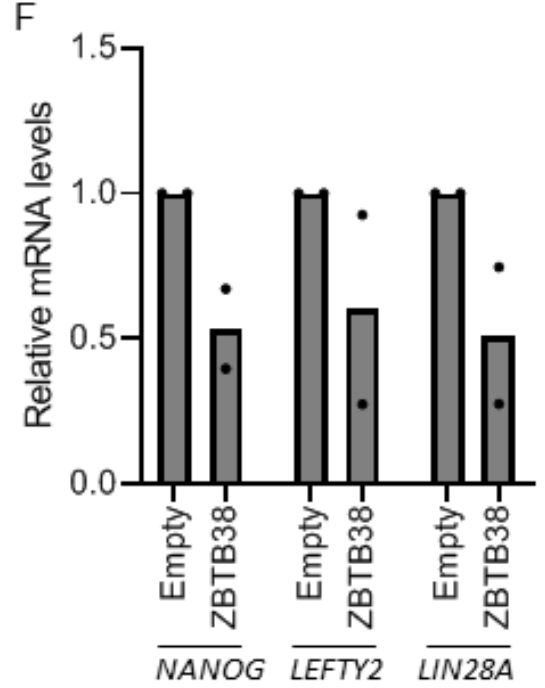

G

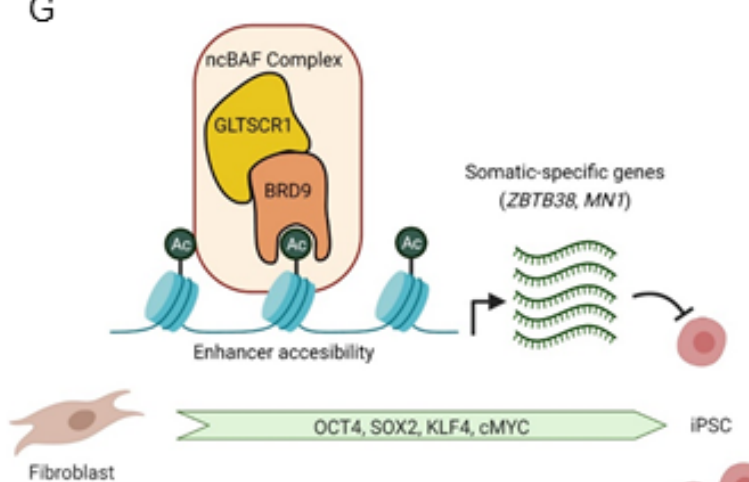

Loss / inhibition of ncBAF complex

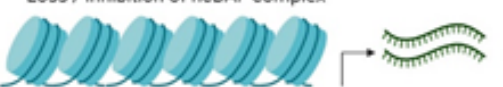


Figure 6. BRD9-regulated MN1 and ZBTB38 act as barrier to reprogramming. (A) Average expression of $M N 1$ and $Z B T B 38$ in TPM across indicated fibroblasts. $\mathrm{n}=3$, biological replicates. p-values were calculated by two sample t-test. * denotes $\mathrm{p}<0.05, * *$ denotes $\mathrm{p}<0.005, * * *$ denotes $\mathrm{p}<0.0005$. Exact $\mathrm{p}$-values for small molecule treatments compared to DMSO control

801 from left to right are: $0.0031,0.0076$ and 0.0002 for $M N 1$ and $0.0089,0.0378$ and 0.0284 for

802 ZBTB38. Exact p-value of BRD9 gRNA expressing cells to control cells is 0.0045 for $M N 1$ and

$803 \quad 0.0026$ for ZBTB38. (B) Fold change in relative MN1 and ZBTB38 mRNA levels upon their

804 overexpression in fibroblasts compared empty vector controls (C) Fold change in the number of

805 TRA-1-60-positive colonies upon MN1 overexpression. Representative well images are above

806 the graph. Bar graphs show the mean. $n=2$, independent biological replicates. (D) Fold change in

807 the number of TRA-1-60-positive colonies upon ZBTB38 overexpression and indicated

808 treatments. Representative well images are above the graph. Bar graphs show the mean and error

809 bars represent standard error of mean. $n=3$, independent biological replicates. $p$-values are

810 calculated by one sample t-test for $m u=1 . *$ denotes $p<0.05$, exact $p$-value is 0.0255 . (E) Fold

811 change in the percentage of TRA-1-60-positive cells on day 6 of reprogramming by comparing

812 ZBTB38 expressing cells to control. Bar graphs show the mean and error bars represent standard

813 error of mean. $n=3$, three biological replicates. $p$-values were calculated by two sample t-test. *

814 denotes $\mathrm{p}<0.05$, exact p-value: 0.0272 . (F) Fold change in relative mRNA levels for the $N A N O G$, 815 LEFTY2 and LIN28 genes on day 6 of reprogramming. Gene expression values were normalized

816 to those observed in empty vector expressing fibroblasts. Bar graphs show the mean and dots

817 indicate two biological replicates. (G) Model for BRD9-containing BAF complex's role in

818 maintaining somatic cell identity during reprogramming. 